\title{
Reconstructing a Tourism Toilet Servicescape Conception Based on Grounded Theory-a Case Study of Lhasa City
}

\author{
Senyao Sang \\ Tibet University, School of Tourism and Foreign Languages, Lhasa, China, 850000 \\ sangkent@126.com
}

Keywords: Tourism toilet; Conceptual framework; Servicescape; Grounded theory; Lhasa

\begin{abstract}
Tourism toilet is an important symbolic in evaluating the regional tourism development. However, related researches about tourism toilet are not sufficient and lack of related concepts of the construction. Therefore, by using qualitative research method, our study reconstructed a conception framework named 'tourism toilet servicescape' grounded by servicescape theory based on Lhasa. Research findings show tourism toilet servicescape has three core themes named 'physical servicescape, social servicescape and cultural symbol servicescape. Each core theme has three secondary themes. Our study is significant on theory and practice of constructing tourism toilet.
\end{abstract}

\section{Introduction}

In recent years, Lhasa City in order to cope with the growing flow of tourists into Tibet, invested a lot of money to enhance the basic public service facilities, including tourism toilets. However, there is no targeted study to explore the Lhasa tourism toilet property composition. Especially in the minority areas of tourism toilets to some extent responsible for the cultural experience of tourists to provide the background of the text of the role of the text and there is little domestic on the regional characteristics of cultural symbols on the development of the importance of tourism research related to the study.

In addition, McKercher divides tourist destinations into three places of travel, non-tourist venues and shared travel venues. Which share the tourist venues for tourists and local residents can also spend the same space. Therefore, in the shared tourist sites, inevitably produce local residents and visitors of the symbolic interaction, to some extent, the impact of the experience of tourists [4]. To Lhasa City Jokhang Temple, for example and its both well-known tourist attractions, but also the local residents pilgrimage space. As a result, space facilities in similar places are, to some extent, responsible for the intercourse between bureaucrats and outsiders. In particular, tourism toilets, for example, tourism, toilet facilities, staff, local residents and tourists, together to build a tourist experience to the toilet environment. However, there are few studies on the current study of tourism toilets, taking into account the specialities of inter-office and inter-city interaction between the using of tourism toilets in shared tourism venues.

In addition, through the study of domestic tourism on the toilet, most of the use of quantitative research techniques (IPA analysis, etc.) or spatial layout of the perspective, the lack of theoretical level from the construction of the depth of tourism latrine exploration.

In summary, this study takes Lhasa as the research background, and takes root in the service environment theory and qualitative research techniques to explore the attribute of tourism latrine. At the same time, this study is different from other studies, taking into account the special nature of shared tourism places to explore the tourism toilet environment, local tourists and tourists' interaction on the perception of the impact of customer service environment.

\section{The Literature Review of Tourism Toilet}

Tourism toilets are defined as "public latrines for tourists who are mainly engaged in tourist 
activities". It includes tourist attractions, tourist venues and related tourist reception sites and all the tourism-related space within the public toilets. Tourism toilet is an important component of tourism public services, not only to meet the basic physiological needs of tourists, but also enhance the overall quality of tourists experience tourism. However, Chinese long-term tourism toilet construction has not played a positive role in enhancing tourist satisfaction. 2014 tourist attractions of the tourists complained that the travel toilets accounted for $23.30 \%$, second only to the ticket price, ranked the most unsatisfactory tourist indicators second place. Therefore, the National Tourism Administration in 2015 launched the "national tourism toilet construction management action" aimed at three years to achieve nationwide tourism destination public toilet standardization.

However, domestic and foreign scholars have not paid enough attention to the study of tourism toilets. While the domestic also in recent years there has been a deep thinking on the construction of tourism toilets.

The domestic literature on tourism toilets can be divided into two types: tourism toilet construction analysis based on tourism public service facilities planning, and tourism toilet quality evaluation analysis based on tourist satisfaction. The paper analyzes the present situation of tourism latrine in Sichuan mountain tourism resort and puts forward the "reasonable layout of tourism toilet", "pay attention to the internal structure of toilet", "pay attention to the unique design of tourism toilet in high altitude area" and "To strengthen the external design of tourism toilets" four-point improvement program. From the macroscopic perspective, the author analyzes the current situation of the distribution of tourism latrine in China.

In the quantitative analysis, Fang Shimin et al. Took the AHP analytic hierarchy process as an example, and evaluated the quality of the tourism toilets in seven aspects: the number, layout, equipment, environment, management, technology and cultural construction of tourism toilet, (Yuelu Mountain, etc.) of the tourism toilet slightly higher quality, and shared tourist sites (railway stations, etc.) the relatively low quality of the results of the toilet. Sun Feng and others analyzed the tourist satisfaction of tourists in Suzhou urban areas. The results showed that the first three elements with the most significant positive impact on the formation of tourists' satisfaction were planning layout, basic functions and management work. The results of IPA analysis of visitors' satisfaction through travel to the main tourist attractions in Shandong show that "toilet odor", "cleaner performance and hygiene", "ergonomic infrastructure", "air circulation" Urgent to solve the toilet properties, it is inferred that some of the scenic areas of Shandong tourism to the existence of the basic physiological needs of tourists has not yet satisfied the lagging situation.

Although domestic research on tourism toilets is gaining attention, the conceptual framework of tourism toilets based on visitor perception is not formed. Bitner builds the concept of servicecape and defines it as a service background that visitors perceive when they receive the service. Tourism latrine as an important part of tourism public services, its physical and cultural, social environment not only affects the visitors to the toilet experience evaluation, but also to some extent affected the overall quality of tourism experience assessment. Therefore, this paper argues that the quality assessment of tourism toilets can be effectively rooted in the concept of service environment.

\section{The Theoretical Background of the Service Environment}

According to the theory of environmental psychology, the visitor's evaluation of the service experience is first influenced by the design and layout of the service environment. Bitner defines the physical layout of the visitor and the staff as a service environment in the service scenario and has been widely used by scholars in various service scenarios over the past 20 years, such as theme parks, festivals.

Bitner believes that the service environment should consider three variables, namely 1) surrounding environmental conditions, including five factors such as temperature, air quality, noise, music and taste; 2) space and function, including layout design, decoration, equipment, etc. It can be seen, Bitner considered the service environment are mostly tangible physical layout and atmosphere, and did not consider the service delivery process of human elements. PZB believes that the quality of service delivery is affected by the service personnel to a considerable extent, and the 
responsiveness, appearance and trustworthiness of the service personnel are recognized and evaluated by the customer as an important component of the service delivery environment. Based on the understanding of the importance of interpersonal elements in the service environment, Rosenbaum reinterprets Bitner's concept of service environment, adds social factors to the original service environment model and defines it as "social servicescape", Which includes "the staff, other customers, social density and other people's emotional show" four dimensions. However, Sang et al. It mentioned that in the context of tourism services, not only service personnel, local residents, other visitors and peers were also perceived by tourists as the service background. Rosenbaum's concept clearly does not take into account the particularity of the tourism service environment, visitors staring at the performance and interaction of local residents is not within the scope of its consideration, the model applicable to the tourism service scene there are some shortcomings.

In addition, Rosenbaum mentions that social symbols can also serve as one of the environmental dimensions that affect the perception of tourists in the service scenario, which defines the social symbolic dimension as a cultural symbol, symbol, and craft. In exploring the national restaurant service environment, Wang also mentions that cultural symbols, one of the service environments, have a significant impact on customer culture's true evaluation and behavioral intentions. Tourism as a tourist to find the "different culture of the true nature of" social activities, different cultural symbol is the perception of its culture is the main signifier. Especially in the ethnic areas to build a consistent national cultural atmosphere for visitors to interpret cultural symbols in depth, experience this has a significant impact.

It can be seen that under the environment of tourism service, the service environment is roughly composed of the dimensions of physical service environment, the dimension of social service environment and the dimension of cultural service environment. However, the concept of service environment of tourism toilets can be fully interpreted by the above three core concepts still exists in the study of the lack of. Therefore, the main purpose of this study is to fill the theoretical gap.

\section{Research Methods}

The purpose of this study is to construct the conceptual framework of tourism toilet service environment through qualitative research method and rooting service environment theory. In the aspect of data collection, this study uses the data collection method of triangulation to ensure the reliability of qualitative research. In the first phase, the researchers developed initial structural interviews through participatory observation and on-site informal interviews.

In the second phase, the researchers conducted a face-to-face interview on visitors to Lhasa's main attractions. The average duration of each interview is around 20-40 minutes and is recorded throughout. This session has a total of 15 interviews. Including 8 men, 7 females, 6 individual, group tourists 9; 20-29 year-old tourists 5, 30-39-year-old tourists 4, 40 years old and above 6 visitors; first visit to Lhasa Visitors 12 people. In order to ensure the privacy of tourists, the analysis process visitors with 1-15 digital code in the form of mark. The recording data is transcribed by five undergraduates of tourism.

In the third stage, based on the preliminary analysis of the contents of the interview, the researchers convened a panel of experts with three tour guides, three tourism professional teachers and two tourism administrative staff members, and conducted an hour-long focus group interview. Interview results are also recorded and transcribed. Interviews are free to discuss the current situation and existing problems of tourism toilets in Lhasa.

The triangular mutual data collection ensures the reliability of the rooted analysis and provides a data base for conceptual construction. Data analysis uses the grounded theory proposed by Corbin and Strauss. This study is based on the service environment theory to construct the conceptual framework of tourism toilet service environment. The core analysis method is open coding secondary concept - the core concept of the three-level induction method. First of all, visitors to the depth of the interview data and focus group interviews data for open coding, followed by its similar content to the secondary concept of the building, and ultimately the formation of the core concept. In the final stage, the researchers examine the results of the deep interview and the results of the 
analysis of the focus group interviews, remove the unreasonable concepts and supplement the concept of missing.

The analysis process is carried out by two tourism professional instructors back to back, the results of the analysis by the third-party tourism professional evaluation, the evaluation results show that two lecturers analysis content similarity reached $96 \%$, qualitative analysis of data reliability requirements of the basic requirements.

\section{Analysis of the Results}

Take Root Analysis Results. Rooted in the theory of service environment, this study has converged three core concepts, namely, physical service environment dimension, social service environment dimension and cultural symbol service environment dimension.

Physical service environment dimension. The physical service environment is the most manageable part of the manager and the dimension that satisfies the most basic perception of the visitor. In this study, the physical service environment is defined as "the physical representation of the environment in which the visitor perceives the environment, the space, the function, the identity, and so on."

The ambient atmosphere refers to the background or atmosphere of the tourist toilets. The atmosphere as a tourist interprets the accompanying text of the present symbol, which has a significant effect on the sensation of the tourists

First, the visualization of the environment, smell, sound and body sensation are intertwined to form a pattern of five-way experience (visual, olfactory, auditory, tactile) directly affect the perception of tourists.

The environmental atmosphere as a secondary concept of the physical service environment, in this study convergence of the five sense of experience (visual, olfactory, auditory, tactile) and clean (physical clean, cultural cleansing) two elements. According to the theory of environmental psychology, the core of the environmental atmosphere is that it provides the outside atmosphere of the visitors 'experience, which can affect the tourists' mood and affect their evaluation of the tourism toilets by changing the cognition of tourists.

Through the analysis of the interview recording, Lhasa tourism toilet service environment in its space and functional elements can be divided into layout, facilities, privacy and security of the four dimensions. The facility is the carrier of service delivery. The integrity, advancement and practicality of the facility are the basic factors that affect the intent of the customer. The facilities of the tourist toilets include both basic functional facilities and additional facilities to facilitate the delivery of basic functions, and even facilities for providing visitors with a short period of leisure and additional facilities for the special population. Travel toilet is not simply to solve the physiological needs of tourists' space, and then evolved into a multi-functional integrated "bathroom (Europe and the United States)", "dressing room ".

In terms of infrastructure, visitors mainly refer to the four basic toilet components (such as "flush equipment", "sink", "floor drain" and "wash basin" (such as "used to sit in the Mainland, suddenly replaced squatting, and I have been in the end of the flushing equipment in the end is to use the foot or hand by hand, although the design looks handy, but good dirty. Focus group interview tour guide 2 also mentioned that the winter wash basin without hot water, many tourists complained to wash their hands with ice water.

Compared to the infrastructure, the most basic functions of the toilet are provided to meet the most basic needs of the tourists. Convenient facilities are a great place for visitors to go to the toilet and go beyond the basic needs. Visitors have expressed the need for convenient conditions such as self-service shopping carts, intertropping hooks / shelters, luggage waiting stations, charging stations, hand dryers and hand sanitizers at the wash basins (eg "outdoors, hygiene products, Toilet paper will not bring a very comprehensive. Put a self-service shopping cart our visitors are also convenient, toilet management can also make money. ". The reason why the tour is different from the daily toilet, is that it can provide convenient services for tourists travel, such as luggage storage services or charging services and water services in the convenience of tourists travel to the toilet at 
the same time also enhance the tourists throughout the trip the sense of happiness

Travel to the toilet for the majority of people to provide functional services at the same time, should also have to meet the short-term needs of tourists happy. The value of tourism public services can be divided into utilitarian values and hedonic values. Tourism toilet is an important part of tourism public service facilities and its value is mainly reflected in the value of the function, that is, tourists on the way to provide a convenient way to solve the physiological needs of the conditions. However, with the increase in the diversification of tourist demand, tourism toilets in the provision of leisure functions to meet the needs of tourists happy value gradually attention. At this stage the country to promote the tourism toilet revolution, has gone beyond the scope of toilet functional standardization, the toilet's versatility and meet the needs of tourists happy to stimulate visitors to experience the experience of creating a positive toilet culture an effective way.

A tag is a physical sign that the service department communicates exactly to the service object. In the tourist toilet service environment, the identifier is a physical carrier that indicates the travel toilets, distinguishes travel toilets and carries on brief description and meaning conveyance. Through the convergence of the contents of the interview, this study from the internal and external identification of the two dimensions of the concept of distinction.

The external marker is a sign that has a distinct distinguishing function from outside the travel toilet. Internal signs are mainly related to the toilet inside the warning signs, publicity slogans and for visitors to wait for the toilet set during the wall hanging LCD screen or a small joke Shorten the tourist waiting time tips and so on. Researchers in the field survey found that most of the tourism halls in Lhasa have obvious warning and publicity slogans, but by setting the LCD screen or tips for short tourists waiting for fatigue is slightly inadequate.

The physical service environment is not only the visitor to interpret the surrounding environment with the text, but also visitors directly to the object of interpretation. The core concept of the physical service environment of this study converges the three sub-concepts of environmental atmosphere, space and function, and identifiers, and constructs the subordinate dimension, which is similar in structure to Rosenbaum and Bitner's service environment.

Social Service Environment Dimension. The social service environment refers to the social elements that visitors perceive during the experience of the toilet. In this study, the core concept includes the performance of the staff, the performance of the local residents and the performance of other visitors.

In view of the performance of the staff, this study draws on the personal ability, responsiveness, friendliness and communication ability and appearance dimension of the staff perceived by the tourists. The performance of the staff is a key factor in the delivery of the service, but also an important dimension of the evaluation of the quality of service. In the focus group interview, the tourism administrative personnel mentioned that most of the sanitation personnel in the tourism toilet in Lhasa are mostly temporary workers. There is a tourist complaint and cannot be dealt with in time for the maintenance of tourism latrine facing the plight.

Lhasa city's tourist attractions are mostly shared travel places, so the process of tourism, tourists and local residents of the interaction stare is inevitable. Some tourists have evaluated the interaction between the tourist toilets and the local Tibetans, mainly in the presence of local Tibetan behavior and the appearance of the signs of gaze, the local Tibetan people's friendly and communication skills.

The performance of other tourists is also an important part of the tourism experience process. In this study, individual respondents mentioned the negative impact of other visitors' performance on their toilets experience in a tourist toilet environment.

Tourism service environment is a typical interpersonal interaction scene. Symbolic interaction takes place in the full space of the tour, including travel toilets. This study captures three types of interactions in the tourist toilets based with staff, locals and other visitors. Sang believes that the interaction in the process of tourism is the process of resource transactions, but also the process of symbolic interaction. While the negative behavior of other tourists has a significant impact on the emotional conflict that triggers the subject. 
Cultural Symbol Service Environment Dimension. The essence of the tourism experience is the process of interpreting and searching for the authenticity of the different cultural symbols. Tourism experience with the overall characteristics of tourists in the interpretation of tourism culture symbols, whether to perceive the consistent culture is really the key to the quality of its evaluation experience. Therefore, tourism public services need to be embedded in the cultural connotation as a whole to meet the overall experience of tourists.

Tourism toilet culture of the true performance is mainly reflected in its appearance, interior decoration and service delivery process of the true performance of three aspects. Appearance, the respondents mentioned the key attractions of Lhasa tourist toilet appearance perfect embedded in the surrounding environment.

Content Analysis Results. Root analysis of the convergence of the three core concepts, nine sub-concepts, while the sub-concepts were summarized under the corresponding lower dimension. The initial open code collected 756 valid base text. In order to analyze the relative importance of tourists' perception of service environment in Lhasa, the researchers analyzed the contents of the basic text. The core concept of view, the physical service environment more than the social service environment more than the cultural symbol service environment. Secondary concept, the first five high-frequency concept of the order of the environment, space and function, staff performance, local residents performance and logo. In the lower dimension, the top five high-frequency dimensions are physical cleaning, infrastructure, vision, smell and staff responsiveness. It can be seen that tourists' cognition of tourism and toilet service environment is still at the level of satisfying their basic needs. The analysis results are consistent with those of Sun Feng et al. and Guo Anxi et al.

\section{Conclusion and Suggestions}

This study aims to establish a conceptual framework for assessing the living environment of tourism toilets by exploring the service environment of tourism and toilet in Lhasa. Therefore, this study mainly adopts the techniques of qualitative research. Rooted in the theory of service environment, the conclusions of this study are as follows.

1) The area of tourism and toilet service in ethnic areas is composed of three core concepts, environmental atmosphere, space and function, identifier, staff performance, local residents 'performance and other tourists' performance, appearance, environment and cultural service environment, social service environment and cultural symbol service environment. Interior decoration and service delivery process of the true performance of the nine sub-concept composition.

2) The results of the content analysis show that the current visitor's awareness of the service environment performance of the tourist toilets is mainly focused on meeting its basic needs, but the versatility of the tourism toilets, the cultural transmission function and the social interaction To some extent affect the visitors to the toilet experience.

In view of the tourism public service department, this study provides the following suggestions for the planning of tourism toilets from the theoretical level.

1) The standardization of tourism toilets should take into account the particularity of its context, in the construction of tourism toilets to give full play to its function as a carrier of cultural significance.

2) Lhasa City, for example, tourism as a shared travel space, staff and local residents and tourists' interaction between the gaze is also the tourism administrative staff need to consider the object. The management of the behavior of tourists, the performance of local residents and improve the quality of service staff should be the focus of tourism toilet management.

3) The basic function of tourism toilets is still the object of concern to tourists. But in improving its basic functions at the same time, increase its versatility for beyond the general tourists to meet, to encourage visitors to have a positive impact.

In this study, qualitative research methods are limited by the amount of specimens, and there are some restrictions on the generalization of research conclusions. And this study mainly in Lhasa City, 
for other areas whether there is a guiding significance is still in doubt. Therefore, future research should use quantitative research techniques to extend the results of this study to a larger population of people in the evaluation, verify whether there is a general. Also in other regions, should not simply apply the results of this study, should consider its regional specificity to re-concept construction.

\section{Acknowledgements}

Fund Project: Tibet University Youth Research and Cultivation Fund Project (ZDPJSK1707).

\section{References}

[1] Zabkar, Brencic, Dmitrovic. Modelling perceived quality, visitor satisfaction and behavioral intentions at the destination level[J]. Tourism Management, 2010, 31: 537-546.

[2] McKercher, Wang, Park. Social impacts as a function of place change[J]. Annals of Tourism Research, 2015, 50:52-66

[3] Choo, Petrick. Social interactions and intentions to revisit for agritourism service encounters[J]. Tourism management, 2014, 40: 372-381

[4] Guo A, Guo Y, Sun X et.al.An empirical research on importance and performance of tourism toilets satisfaction: Based on the comparison of tourists gender[J], Tourism Forum, 2016,9 (4) : 40-49

[5] Sun F, Wang D. Research on present situation and innovation development of tourism toilet construction in China[J]. Resource Development \& Market, 2016, 32（9）:1115-1121

[6] Sun F, Wang D, Niu Y. Influencing factors and innovation of tourism toilet development from the perspective of ecological civilization: Based on tourism satisfaction analysis[J]. Progress in Geography, 2016, 35 (6) : 702-713 